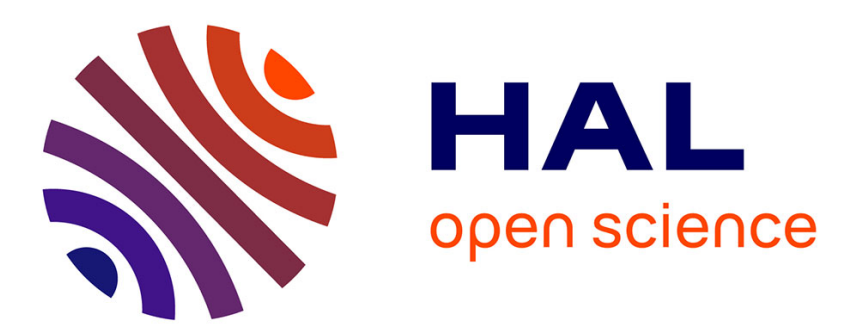

\title{
Novel methods to assess environmental, economic, and social sustainability of main agricultural regions in China
}

Fang Liu, Hongqi Zhang

\section{To cite this version:}

Fang Liu, Hongqi Zhang. Novel methods to assess environmental, economic, and social sustainability of main agricultural regions in China. Agronomy for Sustainable Development, 2013, 33 (3), pp.621633. 10.1007/s13593-012-0131-8. hal-01201374

\section{HAL Id: hal-01201374 \\ https://hal.science/hal-01201374}

Submitted on 17 Sep 2015

HAL is a multi-disciplinary open access archive for the deposit and dissemination of scientific research documents, whether they are published or not. The documents may come from teaching and research institutions in France or abroad, or from public or private research centers.
L'archive ouverte pluridisciplinaire HAL, est destinée au dépôt et à la diffusion de documents scientifiques de niveau recherche, publiés ou non, émanant des établissements d'enseignement et de recherche français ou étrangers, des laboratoires publics ou privés. 


\title{
Novel methods to assess environmental, economic, and social sustainability of main agricultural regions in China
}

\author{
Fang Liu $\cdot$ Hongqi Zhang
}

Accepted: 21 December 2012 / Published online: 25 January 2013

(C) INRA and Springer-Verlag France 2013

\begin{abstract}
In China, dramatic changes of land use since 1980 have induced environmental and socioeconomic problems threatening food security. Therefore, improving sustainability of land use in China is of utmost importance, especially in agricultural regions. So far, few investigations have analyzed sustainability at small scales in China. Here, we propose a methodological framework for assessing the sustainability level of main agricultural regions in China on regional and county levels. We distinguish four sustainability categories: environmental, economic, social, and comprehensive sustainability. Two methods of measuring sustainability were used: (1) the balanced performance method that measures balanced performance among different aspects and (2) the aggregate achievement method that measures aggregate achievement of all aspects. Spatial variation maps of sustainability across counties were produced using a geographic information system, and the limiting factors in each region were identified. Results show that the two methods give highly different values of sustainability levels. The balanced performance method yields lower sustainability values ranging from 0.06 to 0.57 , whereas the aggregate achievement method yields higher sustainability values ranging from 0.11 to 0.87 . Such differences have not been addressed in previous studies. Using the balanced performance method, the Sichuan Basin is the most comprehensive sustainability region with a 0.05 level, while Xinjiang is the least comprehensive sustainability region with a 0.01 level. Using the aggregate achievement method,
\end{abstract}

F. Liu $\cdot$ H. Zhang $(\bowtie)$

Institute of Geographic Sciences and Natural Resources Research, Chinese Academy of Sciences, Beijing 100101, China

e-mail: zhanghq@igsnrr.ac.cn

F. Liu

University of Chinese Academy of Sciences,

Beijing 100049, China the middle reaches of the Yangtze River and Jianghuai region is the most comprehensive sustainability area with a 0.46 level, whereas South China is the least comprehensive sustainability area with a 0.31 level. Sensitivity analysis showed that Songnen and Sanjiang Plains were more sensitive to the indicator selection and aggregation rules. Scale effects were not observed for sustainability assessment at the regional level.

Keywords Sustainability assessment $\cdot$ Land use · Geographic information system · Agricultural regions · China

\section{Introduction}

Food security is a growing concern. Worldwide an estimated 1 billion people lack adequate access to food (Barrett 2010). In China, food security has always been a concern, drawing widespread attention because of the challenging lack of cropland, increasing population, and water shortages (Tao et al. 2009; Godfray et al. 2010). Efficient, sustainable use of land can meet the food demands of even huge societies and guarantee national food security.

Since the reform and opening up that took place in China in the 1980s, China's cropland has experienced great changes, but this has been threatened by several environmental and socioeconomic issues. Most of the lost cropland was of good quality and highly productive, but most of the new cropland is located in places with poor soil fertility and limited water resources (Liu et al. 2005). This has not been beneficial for food security. The newly added cropland was mainly reclaimed from woodlands and grasslands in northeastern and northwestern China, where the ecological environment is fragile (Liu et al. 2005). This causes considerable land degradation and environmental deterioration (Jiang et 
al. 2003; Liu et al. 2009). Also, critical conflict between population growth and scarce land resources, soil pollution, and other problems are increasingly common, especially in eastern coastal provinces (Yang 2001). In this way, the sustainability of land use in China, especially cropland, is becoming increasingly important. An increasing number of studies on land use sustainability assessment in China have been published (Wu and Huang 2001; Kong and Ou 2006). However, none of them have provided an overall view of the main agricultural regions across China. Most of these studies have been performed at the province or city level, leaving detailed spatial discrepancies with respect to land use sustainability difficult to characterize at finer scales.

There is a general agreement that sustainable development includes environmental, economic, and social dimensions (Cai and Barry 1994; Van Cauwenbergh et al. 2007). Sustainability indicators characterizing these three dimensions are generally used to bridge the gaps between theoretical concepts and actual measures (Bell and Morse 2008; Gómez-Limón and Riesgo 2009). Because individual indicators are usually inadequate to represent the overall sustainability of a complex system, indicator integration has been increasing recognition as a useful method of analyzing complex multidimensional issues (Andreoli and Tellarini 2000; Meul et al. 2008). Multi-criteria methods are used to assess sustainability. These include multi-attribute utility theory, the Electre method, and the smarter method et al. (Guitouni and Martel 1998; Malczewski 2006).

China has eight main agricultural regions (National Agricultural Regionalization Commission 1981). These are the Sichuan Basin (I), Eastern Inner Mongolia (II), Xinjiang (III), Middle reaches of the Yangtze River and Jianghuai region (IV), Sanjiang Plain (V), Huang-HuaiHai Plain (VI), South China (VII), and Songnen Plain (VIII; Fig. 1). These areas occupy an important position in Chinese agriculture. Cropland in these regions accounts for more than $50 \%$ of the total cropland in China, and agricultural products account for over $55 \%$ of the total production in China. The aim of this study was to establish an index system for sustainability assessment at the county level and assess the sustainability of land use in China's eight main agricultural regions from environmental, economic, and social perspectives. Using two different methods of measuring sustainability, indicators were aggregated with a noncompensatory approach (minimum value approach) and a compensatory approach (multi-attribute value theory). We determined and compared the sustainability of land use among China's main agricultural regions, and investigated spatial discrepancies and restraints in these regions. The limiting factors in each region were identified for future reference. Finally, scale effects and sensitivity analysis were discussed.

\section{Materials and methods}

\subsection{Conceptual framework}

Sustainable land use in agricultural regions means using land assets in a way that maintains or enhances productivity, protects the potential of natural resources, prevents degradation, meets increasing demands for fresh and healthful food, maintains the economic viability of agricultural production, and accomplishes all of the aforementioned in a manner acceptable to society. Environmental, economic, and social conditions were used to determine whether land use management was sustainable, would become so, or would remain so. In this study, sustainability was measured in two ways: measurement of balanced performance among different aspects and measurement of the aggregate achievement of all aspects.

Because sustainability issues are multidimensional, we evaluated environmental, economic, and social sustainability. Environmental sustainability indicates the protection and sustainable use of natural resources, especially water and soil resources, without pollution or degradation. Agricultural practices may cause resource shortages due to extensive use, and environment pollution can be a side effect of pesticide overuse. Many of these negative impacts can be minimized. Economic sustainability involves the maintenance and increase of financial profitability. The intensity of land use management may contribute to financial viability. This is because the availability of financial resources influences the land use management techniques and practices chosen by farmers, such as the degree of input intensity. High gross output values of farming can contribute to economic sustainability. Social sustainability involves a stable and prosperous society, in which the majority of individuals have access to sufficient food.

\subsection{Indicators selection and design}

\subsubsection{Indicator selection}

A set of indicators was used to measure sustainability of land use system from three perspectives: ecological sustainability, economic sustainability, and social sustainability. Several criteria were considered during the construction of these indicators: (1) measurability: indicators were required to be measurable over space and quantified using simple techniques; (2) relevance to sustainability: an obvious relationship had to exist between all indicators and sustainability; (3) dominance: more important and influential indicators were preferred; (4) universality: in views of the large size of the study area and its diversity with respect to natural and economic conditions, indicators had to be 
Fig. 1 Spatial variation map of the degree of sustainability for agricultural regions.

Sustainability was divided into five ratings by Jenks natural break optimization: very low, low, medium, high, and very high. The various regions are indicated as follows: I Sichuan Basin, II Eastern Inner Mongolia, III Xinjiang, $I V$ Middle reaches of the Yangtze River and Jianghuai region, $V$ Sanjiang Plain, VI Huang-HuaiHai Plain, VII South China, and VIII Songnen Plain

\section{a Balanced performance}

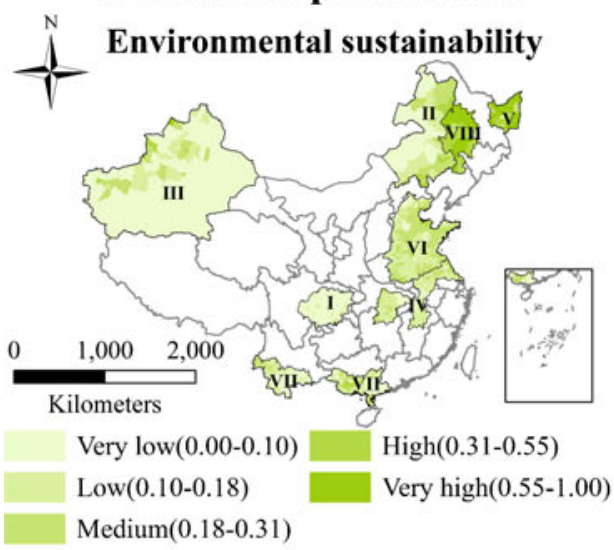

Economic sustainability
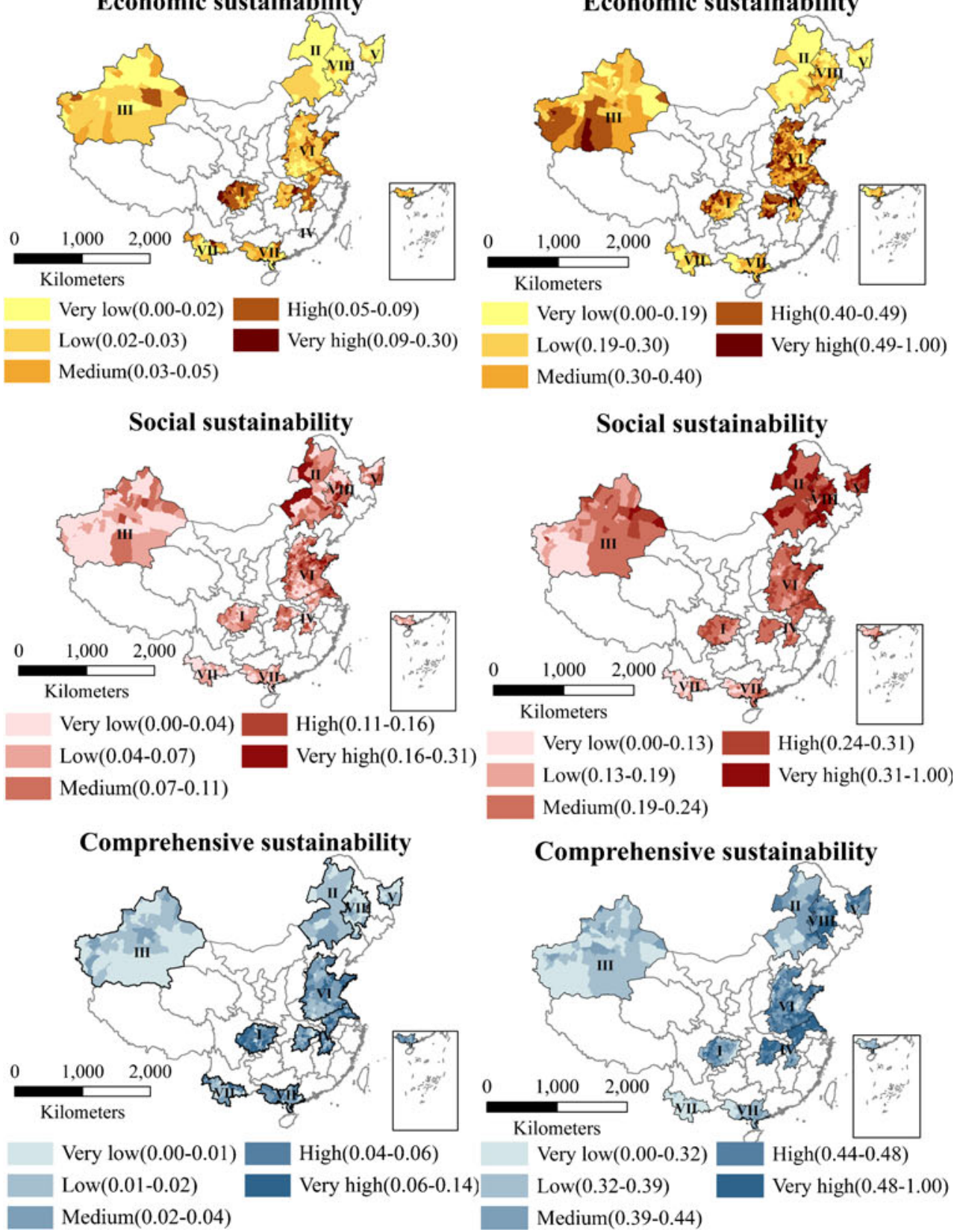

b Aggregate achievement

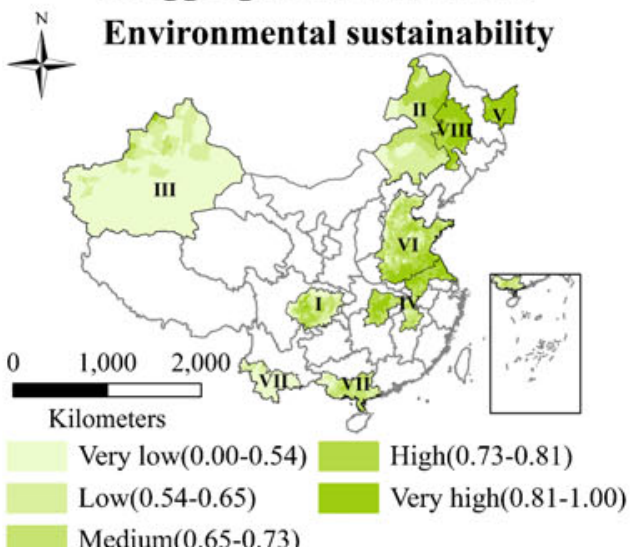

Economic sustainability

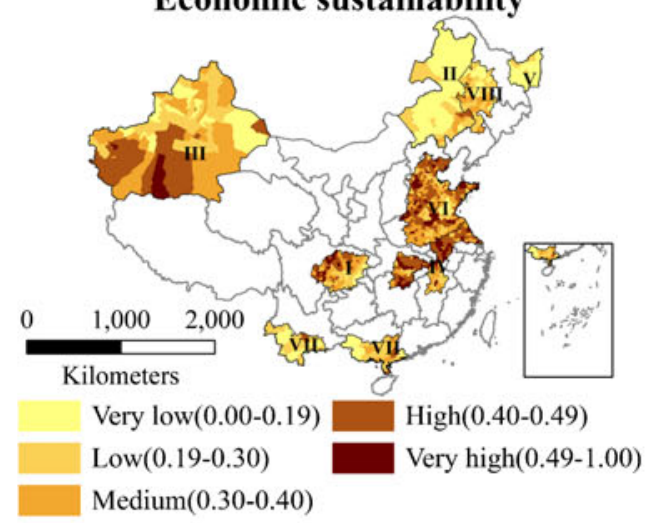

Social sustainability

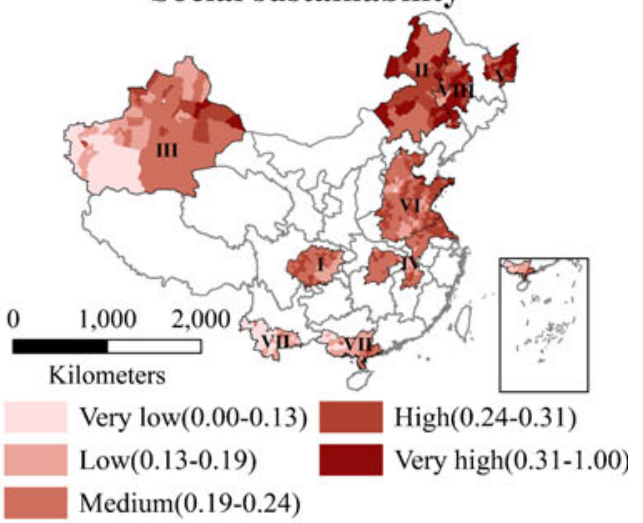

Comprehensive sustainability

Medium(0.39-0.44) 
suitable for every region; and (5) obtainability: data had to be accessible for all regions.

Based on extensive review of the literature and regional characteristics of the areas to be studied, we identified 14 indicators suitable for assessing sustainability framework at the county level. For each indicator, concise definitions, methods of calculation, and indicator type were shown in Table 1.

\subsubsection{Indicator validation}

Indicator validation is an essential process affecting the quality, reliability, utility, and objectivity of sustainability assessment (Roy and Chan 2012; Bockstaller and Girardin 2003). According to Meul et al. (2009), indicator validation involves two key aspects: an evaluation of the indicator's accuracy and an evaluation of its credibility. In this study, we consider only the former aspect because credibility evaluation involves end-use validation, which is not feasible for studies of large areas. For indicator's accuracy, two validation aspects can be considered: design validation and output validation (Bockstaller and Girardin 2003). A panel of experts from the Chinese research community (Department of Land Cover Change and Land Resource Research, Institute of Geographic Sciences and Natural Resources Research) was invited to evaluate the scientific quality of the construction of the indicators and the indicator output for the purpose of evaluating their reliability.

\subsubsection{Data collection}

Natural resource data including land use maps of China in 2000 and 2008, land resource maps of China $(1: 1,000,000)$, and soil type maps of China were obtained from the Data Center for Resource and Environmental Sciences of the Chinese Academy of Sciences. Socioeconomic data were mainly obtained from official statistical data in 2009, including statistical yearbooks, statistical bulletins, rural statistical yearbooks, and financial yearbooks of the relevant provinces and cities.

\subsection{Indicators aggregation}

According to Nardo et al. (2008), the aggregation of indicators includes three steps.

\subsubsection{Normalization}

Indicators were categorized into three types: (a) positive indicators, representing higher sustainability as they increase in value; (b) negative indicators, representing lower sustainability as they increase in value; and (c) doublemeaning indicators, being positive indicators under a threshold and negative indicators above the threshold; the only indicator in this study that was included in this category was the chemical fertilizer consumption since the marginal product of fertilizer is positive for increasing grain production until it approaches the optimum level, and then it becomes negative when fertilizer levels rise above the optimum; in this latter case, fertilization leads to ecological risk without increasing production.

The normalization of an indicator $j$ of the ist county $\left(R_{i j}\right)$ was calculated using the following equation:

$R_{i j}^{\prime}=1-\frac{R_{j}^{*}-R_{i j}}{R_{j}^{*}-R_{*_{j}}}=\frac{R_{i j}-R_{*_{j}}}{R_{j}^{*}-R_{*_{j}}}$

$R_{i j}^{\prime}=1-\frac{R_{i j}-R_{j}^{*}}{R_{*_{j}}-R_{j}^{*}}=\frac{R_{*_{j}}-R_{i j}}{R_{*_{j}}-R_{j}^{*}}$

Where $R_{i j}^{\prime}$ is the normalized value of $R_{i j} ; R_{j}^{*}$ is the optimum value of the indicator $R_{i j}$; and $R_{* j}$ is the worst value achieved by the $j$ th indicator of sustainability. When $R_{i j}$ belongs to the type (a)/(b), $R_{j}^{*}$ represents the maximum/ minimum value, $R_{*_{j}}$ is the minimum/maximum value, and equations (3) and (4) are used on $R_{i j}$ and $R_{* j}$, respectively. In the case of the double-meaning indicator (c), the value of $R_{j}^{*}$ is determined using the method described below. Equation (3) is used if this value is below $R_{i}^{*}$, and Eq. (4) is used if it is above $R_{j}^{*}$.

The optimum level of fertilizer was calculated by means of the fertilizer response function that reflected the relationship between fertilizer levels and yield levels by means of fitting models. In this paper, the level of fertilizer that corresponded to peak grain yield was considered the optimum level of fertilizer. This assumption was based on a quadratic curve model built for each region (Jin 1983), due to significant variation in land quality among regions.

\subsubsection{Weighting}

The Analytic Hierarchy Process (AHP) was employed to determine factor weight (Table 1). If the consistency index/ random index $(\mathrm{CI} / \mathrm{RI})$ ratio is smaller than 0.10 , the degree of consistency is satisfactory and AHP will yield meaningful results. The CI/RI ratio in this study was less than 0.10 , indicating an appropriate use of AHP.

\subsubsection{Aggregation}

In the case of the measurement focusing on aggregate achievement, indicators were aggregated using multiattribute value theory, a compensatory method, because of its ability to analyze many dimensional conditions and allow 


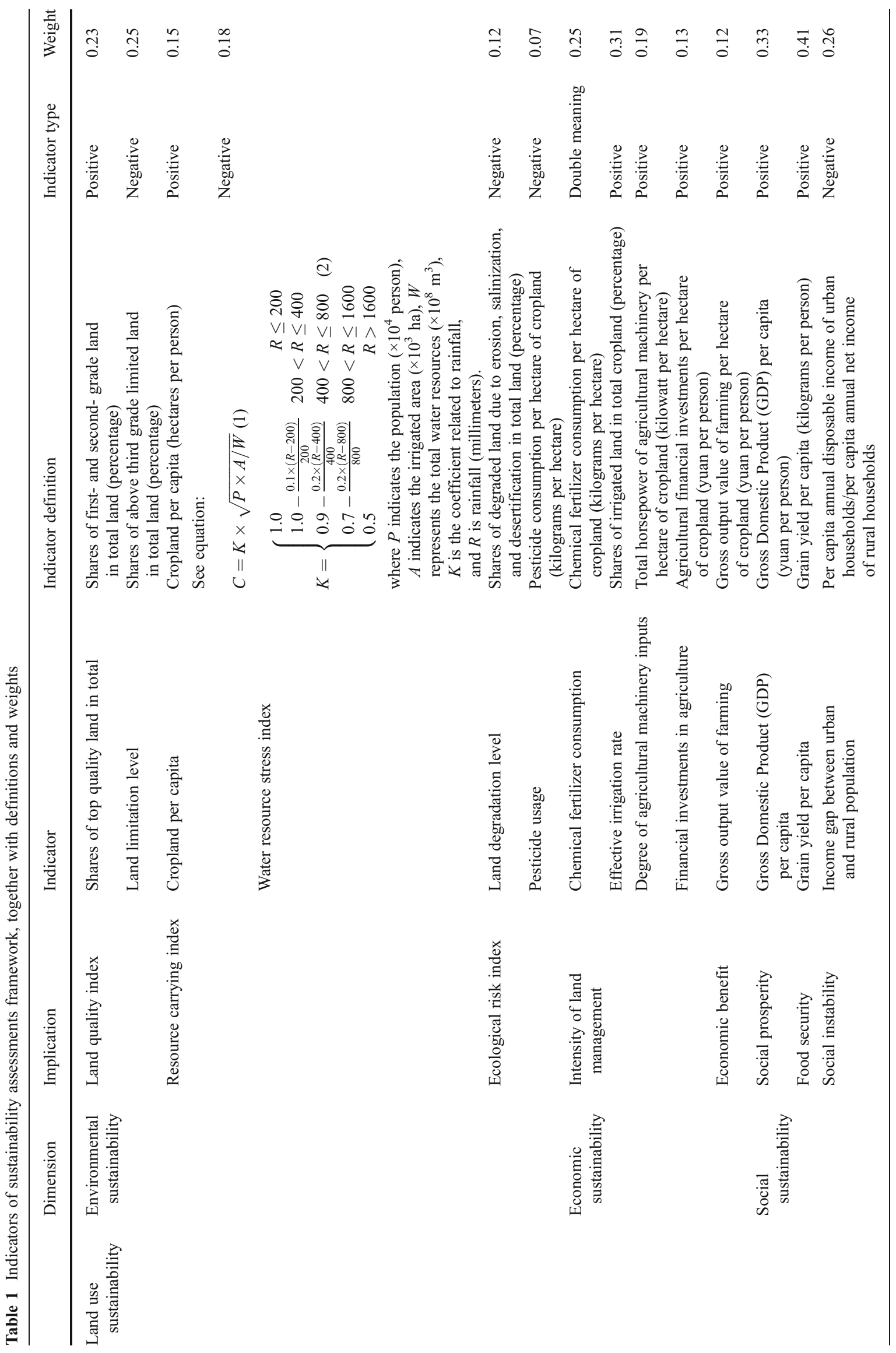


the conduction of assessments (Dantsis et al. 2010). The value function of the additive model was used because of its simplicity.

$C I_{c}=\sum_{j=1}^{\mathrm{n}} w_{j} v_{j}\left(x_{j}\right)$

Where, $\mathrm{CI}_{c}$ is the comprehensive sustainability of county $c, w_{j}$ is the weight of the sustainability indicator $j$ estimated by AHP, $n$ is the number of indicators, and $v_{j}\left(x_{j}\right)$ transforms individual indicator $x_{j}$ into commensurable units between 0 and 1 , and the distance to a reference method was applied (see Section 2.3.1).

For the second measurement of sustainability, which focuses on balanced performance among different aspects of sustainability, a minimum value method (minimum aggregation method) was used.

$C I_{c}=\operatorname{Min}_{j=1}^{n} v_{j}\left(x_{j}\right)$

Based on the three dimensions of sustainability evaluated here (environmental, economic, and social), which were calculated separately, the final goal of land use sustainability was here considered the comprehensive sustainability degree of three components which were considered equally important, as dictated by the definition of sustainability (Van Passel et al. 2007).

\subsection{Scaling up from county level to regional level}

Sustainability degree calculated at county level is scaled up at regional level through a weighted mean (Eq. 7).

$C I_{r}=\frac{\sum_{c=1}^{t}\left(C I_{c} \cdot s_{c}\right)}{\sum_{c=1}^{t} s_{c}}$

Where, $\mathrm{CI}_{r}$ is the region-level comprehensive sustainability of region $r, s_{c}$ is the area of the $c$ th county, and $t$ is the number of counties in region $r$.

\subsection{Data analysis}

\subsubsection{Statistical analysis}

The Kruskal-Wallis test is a nonparametric test used to compare three or more independent groups of sampled data, which makes no assumptions about data distribution (TexaSoft 2011). Sample data in this study did not meet the homogeneity of variance test, so the Kruskal-Wallis test was selected to compare average degrees of sustainability among the eight regions under analysis. The Spearman correlation coefficient is a nonparametric measure of statistical dependence between two variables with no assumption of normal distribution for data, so it was used for sensitive analysis.

\subsubsection{GIS analysis}

Geographic information system (GIS) software is increasingly used among regional managers for storing data and producing maps. Here, GIS was used as the platform for generating, displaying, and spatially analyzing information for the measurement of sustainability.

\subsubsection{Spatial variability map}

To produce the spatial variability maps of sustainability at the county level, the degree of sustainability was divided into five ratings (very low, low, medium, high, and very high; Fig. 1) in Arcmap by Jenks natural break optimization. Table 2 presents descriptive statistics of the degree of sustainability by five ratings according to different methods of measuring sustainability.

\section{Results and discussion}

3.1 Comparative analysis of the sustainability level of agricultural regions and spatial variations within each region

The Kruskal-Wallis test was performed to compare average degrees of sustainability among the eight regions under analysis, indicating a significant difference among the eight groups at the 0.05 significance level. The sustainability ranking of the eight agricultural regions varied with different methods of measuring sustainability. An analysis and discussion of the two cases were provided in the next two sub-sections.

\subsubsection{Sustainability measurement focusing on balanced performance among different aspects}

In terms of comprehensive sustainability (Fig. 1a), the Sichuan Basin was the most sustainable region with value of 0.05 , followed by the middle reaches of the Yangtze River and Jianghuai region and Huang-Huai-Hai Plain. Xinjiang was the least sustainable region with a value of 0.01 .

The most environmentally sustainable region was the Songnen Plain, followed by the Sanjiang Plain. In these two areas, 96.15 and $85.71 \%$ of counties, respectively, showed medium or higher levels of sustainability. The region with the lowest level of sustainability was Xinjiang (Fig. 1a). 
Table 2 Mean and standard deviation of the degree of sustainability by ratings with respect to environmental, economic, social, and comprehensive sustainability

\begin{tabular}{|c|c|c|c|c|c|}
\hline Methods & Ratings & $\begin{array}{l}\text { Environmental } \\
\text { sustainability }\end{array}$ & $\begin{array}{l}\text { Economic } \\
\text { sustainability }\end{array}$ & $\begin{array}{l}\text { Social } \\
\text { sustainability }\end{array}$ & $\begin{array}{l}\text { Comprehensive } \\
\text { sustainability }\end{array}$ \\
\hline \multirow[t]{5}{*}{ Balanced performance } & Very low & $0.06 \pm 0.03$ & $0.01 \pm 0.01$ & $0.03 \pm 0.01$ & $0.01 \pm 0.00$ \\
\hline & Low & $0.14 \pm 0.02$ & $0.03 \pm 0.00$ & $0.06 \pm 0.01$ & $0.02 \pm 0.00$ \\
\hline & Medium & $0.21 \pm 0.03$ & $0.04 \pm 0.01$ & $0.09 \pm 0.01$ & $0.03 \pm 0.00$ \\
\hline & High & $0.41 \pm 0.07$ & $0.07 \pm 0.01$ & $0.13 \pm 0.01$ & $0.04 \pm 0.01$ \\
\hline & Very high & $0.68 \pm 0.08$ & $0.11 \pm 0.04$ & $0.20 \pm 0.04$ & $0.07 \pm 0.01$ \\
\hline \multirow[t]{5}{*}{ Aggregate achievement } & Very low & $0.48 \pm 0.05$ & $0.13 \pm 0.04$ & $0.10 \pm 0.02$ & $0.29 \pm 0.03$ \\
\hline & Low & $0.61 \pm 0.03$ & $0.25 \pm 0.03$ & $0.16 \pm 0.02$ & $0.36 \pm 0.02$ \\
\hline & Medium & $0.69 \pm 0.02$ & $0.35 \pm 0.03$ & $0.22 \pm 0.01$ & $0.41 \pm 0.02$ \\
\hline & High & $0.77 \pm 0.02$ & $0.44 \pm 0.02$ & $0.27 \pm 0.02$ & $0.46 \pm 0.01$ \\
\hline & Very high & $0.85 \pm 0.04$ & $0.53 \pm 0.04$ & $0.36 \pm 0.05$ & $0.50 \pm 0.02$ \\
\hline
\end{tabular}

The highest ranked region for economic sustainability was the Sichuan Basin, followed by the middle reaches of the Yangtze River and Jianghuai region. Spatial variation was also observed within agricultural regions (Fig. 1a). In the Sichuan Basin, the best counties from the economic sustainability viewpoint were located primarily in Chengdu, Mianyang, Leshan, and Yaan. The Songnen Plain and Sanjiang Plain were the two lowest ranked regions, with 71.15 and $80.95 \%$ of the counties showing low degrees of economic sustainability.

Eastern Inner Mongolia and the Songnen Plain were the two highest ranked regions with respect to social sustainability (Fig. 1a). The two regions with the lowest levels of social sustainability were Xinjiang and South China; spatial variation showed that counties in northern and eastern Xinjiang were more sustainable than those in southern and western Xinjiang.

\subsubsection{Sustainability measurement focusing on aggregate achievement among all aspects}

The second method produced different results. The highestranked region was the middle reaches of the Yangtze River and Jianghuai region with respect to comprehensive consideration of all three aspects of sustainability, and South China showed the lowest level of sustainability under the comprehensive sustainability system (Fig. 1b).

The Sanjiang Plain and Songnen Plain were found to be the most environmentally sustainable regions, with more than $70 \%$ of counties showing a high degree of sustainability. The two regions with the lowest sustainability were found to be South China and Xinjiang (Fig. 1b). Remarkable variations existed in both regions (Fig. 1b). Degrees of sustainability in western Guangxi and eastern Guangdong were found to be higher than those of southwestern Yunnan. Counties with higher degrees of sustainability were mainly distributed in cropland concentration areas such as the Yili River valley, Manasi River irrigation area, Irtysh River valley, and Emin River basin.

With respect to economic sustainability, the highest ranked regions were the middle reaches of the Yangtze River and Jianghuai region and Huang-Huai-Hai Plain (Fig. 1b). Five high-sustainability zones were found in the Huang-Huai-Hai Plain: Jiaozhou peninsula, Beijing-Tianjin municipal circle, the Baoding and Shijiazhuang, ShangqiuXuzhou- Jining zone, and the Liaocheng-Puyang-AnyangJiaozuo belt. The counties with relatively low degrees of economic sustainability within the middle reaches of the Yangtze River and Jianghuai region were primarily in Jiangxi Province (Fig. 1b). The lowest level of economic sustainability was observed in the Sanjiang Plain, in which all the counties failed to reach even a medium degree of sustainability.

The Sanjiang Plain, Eastern Inner Mongolia, and Songnen Plain turned out to be more sustainable in social respects, with 100,100 , and $92.31 \%$ of counties showing a medium or higher degree of sustainability (Fig. 1b). The lowest ranked region was South China, and counties in western Guangxi and eastern Guangdong exhibited higher sustainability levels than southwestern Yunnan (Fig. 1b).

\subsection{Analysis of restraints in each region}

Identifying the restraints in each region is an important job, which could provide references for regional managers to make better decisions. In this study, we assumed that if the average value of one indicator in a region was smaller than any other region and lower than national average, the indicator was considered a restraint for that region. 


\subsubsection{Environmental sustainability}

\section{Land quality}

Eastern Inner Mongolia and Xinjiang were weak in land quality. Much of the land in these two regions was infertile and plagued by erosion and degradation. More than $90 \%$ of counties in both regions were below the national average with respect to land quality.

\section{Resource carrying capacity}

Conflicts between the population and cropland resources are intense in the Sichuan Basin. All the counties are below the national average in cropland per capita. Urbanization and population growth were considered the greatest contributors. Over the period from 2000 to 2008, cropland declined in area by $1.96 \%$, while population increased by $10.15 \%$. Among the land transformations from cropland to other uses, urbanization accounted for the largest share, showing $66.49 \%$.

As the second largest grain producer in China, the Huang-Huai-Hai Plain faced the most severe problem of water shortage. Huang-Huai-Hai is home to $39.4 \%$ of China's cropland and $34.7 \%$ of its population but only $7.7 \%$ of its water resources. Water resources per capita are only $449 \mathrm{~m}^{3}$. Water resource stress index showed that Huang-Huai-Hai Plain had the largest water resource stress, $98.65 \%$ of counties suffering from high degree of water scarcity. This caused the overexploitation of surface water and groundwater to meet industrial and agricultural demands, resulting in lowering of groundwater tables, formulation of sink holes, and heavy pressure on irrigated agriculture (Shu et al. 2001).

\section{Ecological risk}

Overuse of pesticides can cause a wide range of environmental impacts (Boyd 2001). Pesticide usage was more intense in developed coastal areas, such as the middle reaches of the Yangtze River and Jianghuai region and South China. Pesticide usage in 84.21 and $72.92 \%$ of counties in these regions were above the national average.

Xinjiang and Eastern Inner Mongolia were found to be the most fragile regions from an ecological point of view. Both are plagued by serious land degradation. From 1990 to 2008, the net increment of cropland was $126.81 \times 10^{4} \mathrm{hm}^{2}$ in Xinjiang and $116.77 \times 10^{4} \mathrm{hm}^{2}$ in Eastern Inner Mongolia. New cropland exacerbated the problems of water shortage and worsened the situation of land degradation because excessive use of upstream water for irrigation has progressively reduced the volume and quality of water available to areas downstream, causing a series of eco-environmental problems such as land desertification (Jiang et al. 2003).

\subsubsection{Economic sustainability}

Sichuan Basin has a low level of agricultural mechanization, with $55.37 \%$ of its counties below the national average. This is largely dependent on the fact that $83.07 \%$ of the total cropland area is very steep.

The amount of irrigated land in the Songnen and Sanjiang Plains is limited, with 31.01 and $12.88 \%$ of the total cropland under irrigation, respectively. These areas have relatively abundant water resources but a scarcity of cropland water infrastructure. The only minimal investment in irrigation has caused poor crop resistance to disasters, creating significant instability and reductions in yield.

Fertilizer usage should be properly controlled in South China to prevent overuse (average fertilizer usage, $656.29 \mathrm{~kg} / \mathrm{ha}$ ). South China has many mountains and hills, so large-scale mechanization is difficult to implement. Consequently, over $80 \%$ of its counties were found to be below the average national level with respect to the use of agricultural machinery.

\subsubsection{Social sustainability}

When assessing social sustainability, South China performed poorly in grain production, showing per capita production below the national average. This has happened because priority was given to cash crops instead of grain crops. Reducing the income gap between urban and rural areas is crucial to the maintenance of social stability.

\subsection{Discussion}

\subsubsection{Two different methods of measuring sustainability}

Two different methods were used to measure whether specific land use systems were sustainable or likely to remain so in the future. In the framework of these two methods, land use sustainability was assessed, and different rankings and variations appeared among and within agricultural regions. Under the first method, which focused on the balanced performance among different aspects of sustainability, the weakest aspect of sustainability was taken to represent the overall sustainability level of the area in question. This information may be helpful in explorations of the limiting factors that hinder sustainable land use. Comprehensive sustainability based on more balanced conditions was found to 
be higher in the Huang-Huai-Hai Plain than on the Songnen Plain. This was largely attributable between differences in the balance among environmental, economic, and social dimensions. Under the second method, which places more attention on aggregate performance among all aspects, the sustainability assessment permits compensation between indicators, that is, a deficit in one indicator could be offset by a surplus in another. The Songnen Plain, when viewed using aggregate performances, was shown to be more sustainable than the HuangHuai-Hai Plain despite its lower sustainability level in economic dimension but made up for it with higher performances in the other dimensions.

\subsubsection{Indicator selection}

Indicators selected varied across sustainability assessment studies performed by different scholars, who had various aims and worked in different study areas. In this paper, indicators selected for land use sustainability assessment placed attention on agricultural aspects of land use, mainly including the input and output conditions of agricultural land. This may not be the case in other regions, but in particular for the eight regions evaluated here with a long history of agricultural use and important positions in Chinese agriculture. Polices that affect land use play an important role in sustainable land use management. However, we failed to select corresponding indicators due to the difficulty in quantification of the impact of polices. But it will be addressed in future studies.

\subsubsection{Scale effects}

Scale is an important and inevitable issue in the assessment of sustainability. In this paper, emphasis was placed on evaluating whether the geographical scale has any influence on the outcomes of sustainability. The impact of geographic area on the degree of sustainability was calculated and explored at both the regional and county levels.

Considering that regions differ in size and include different numbers of counties, the relationships between the degree of regional sustainability and numbers and sizes of counties were evaluated. As shown in Fig. 2A, the degree of sustainability of eight regions showed no significant relationship with county area or number of counties with respect to environmental, economic, social, or comprehensive sustainability. In this way, no evidence for scale effects in determining the degree of regional sustainability was found.

Scale effects were also explored within each region at the county level. Considering the similar natural and socioeconomic conditions within a region, scale effects at the county level might be more measurable. The relationship between the overall sustainability and county area for each region is shown in Fig. 2B. Except for the Sichuan Basin, no apparent features appeared for any of the regions. For the Sichuan Basin, degree of sustainability was found to decrease when county area increased, indicating a scale effect on the degree of sustainability at the county level. In conclusion, while scale affected the degree of sustainability in Sichuan Basin at the county level, changes in scale, in terms of county area and number of counties, had little repercussions on the results of sustainability assessment at the regional level.

\subsubsection{Sensitivity analysis}

Some subjective judgments are inevitably involved in the process of sustainability assessment. So a good modeling practice requires the evaluation of the confidence in the model and assessment of the uncertainties and sensitivity analysis (Nardo et al. 2008). The validity of the sustainability assessment system developed here was assessed by evaluating its sensitivity to three main sources of uncertainty: the number of indicators in the data set, the weights of the indicators, and the aggregation rule.

The impact of excluding a single indicator from the indicator framework on the region ranking of sustainability level was evaluated. Indicators were aggregated using an additive model. Equal weights for indicators within each dimension and equal weights for each of the three dimensions were maintained to control the effects of weighting on the results. The distance to a reference method was used for the normalization before aggregation. Fifteen scenarios were analyzed, one with the entire set of 14 indicators, and 14 sets of 13 indicators each. Figure 3 a provides statistics for the regions rank range, that is, the difference between the worst and the best-case scenarios. Results showed the more sustainable the regions are, the more sensitive to the indicator selections. The wide rank ranges were observed in Sanjiang Plain, Songnen Plain, Huang-Huai-Hai Plain, and the middle reaches of the Yangtze River and Jianghuai region due to several indicators. To be more specific, the Sanjiang Plain and Songnen Plain were both sensitive to 'grain yield per capita'. The HuangHuai-Hai Plain was sensitive to 'shares of top quality land in total', and the middle reaches of the Yangtze River and Jianghuai region were found to be sensitive to 'water resource stress index'.

Next, the impact on the ranking of the weights to be assigned to the indicators was studied. Three weighting 
Regional level

a Balanced performence

b Aggregate achievement
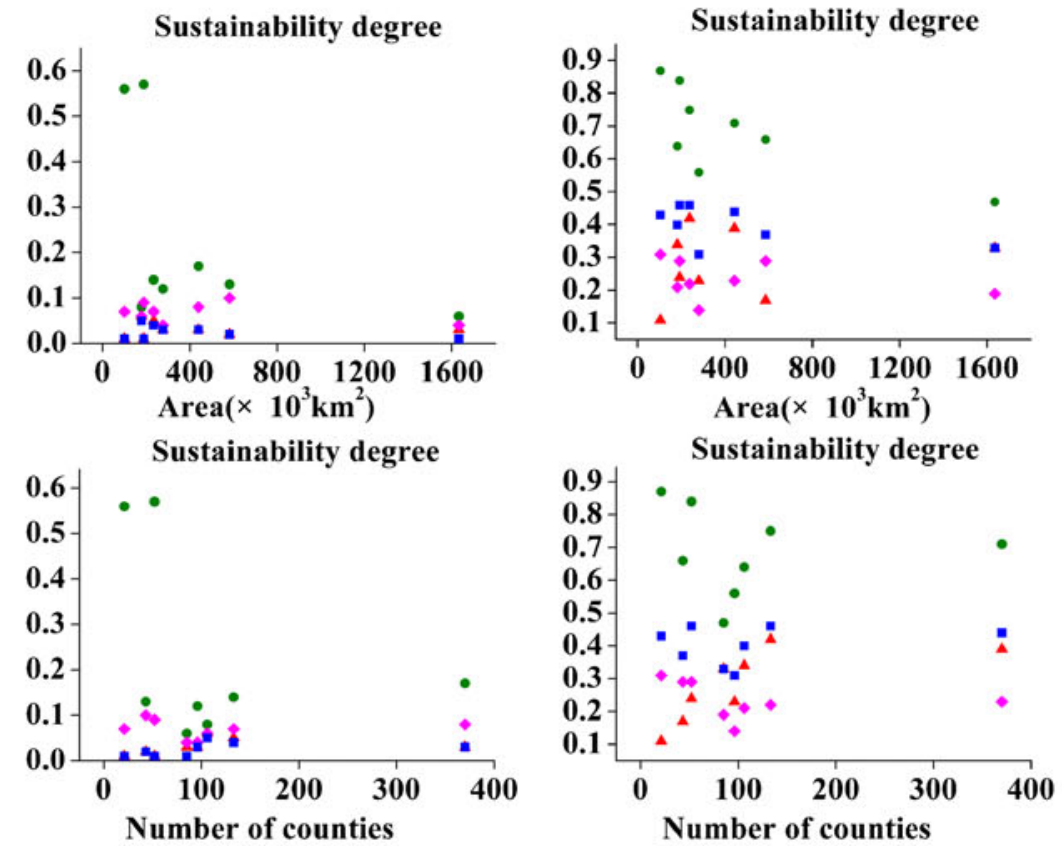

B

\section{a Balanced performence}
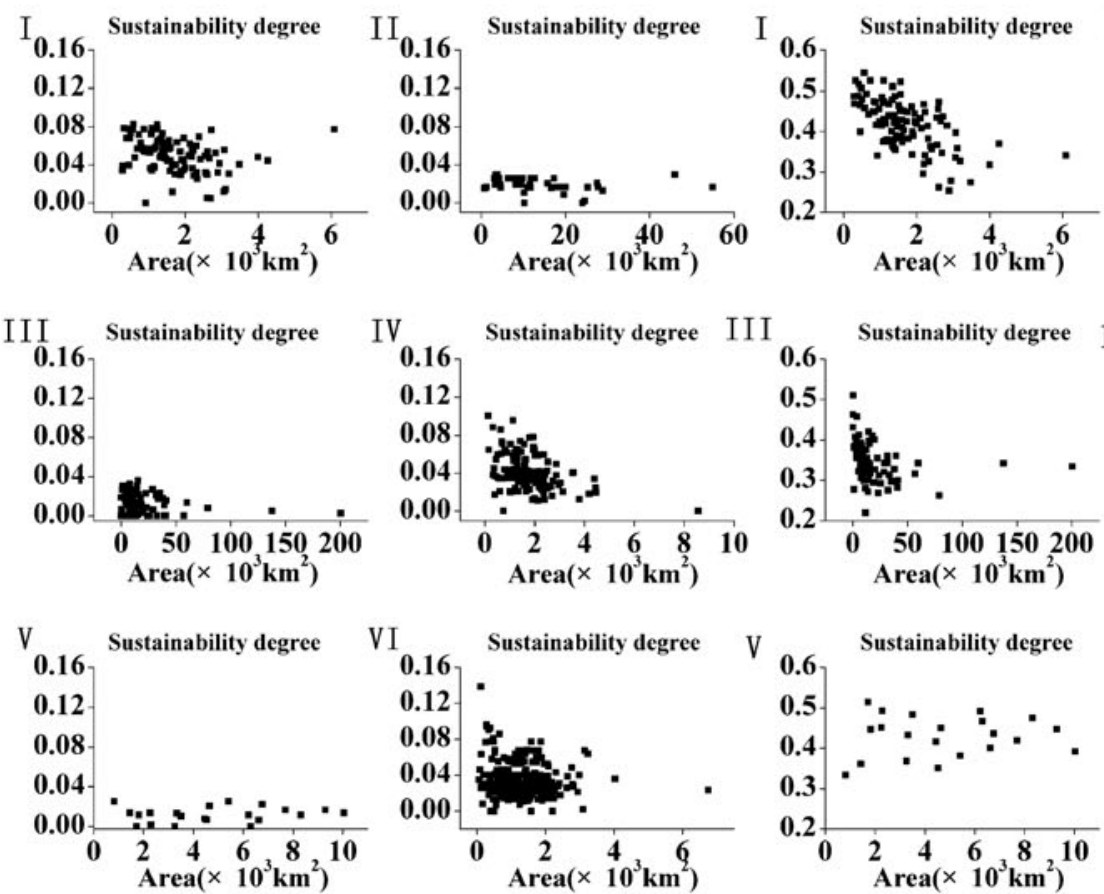

b Aggregate achievement

\section{County level}
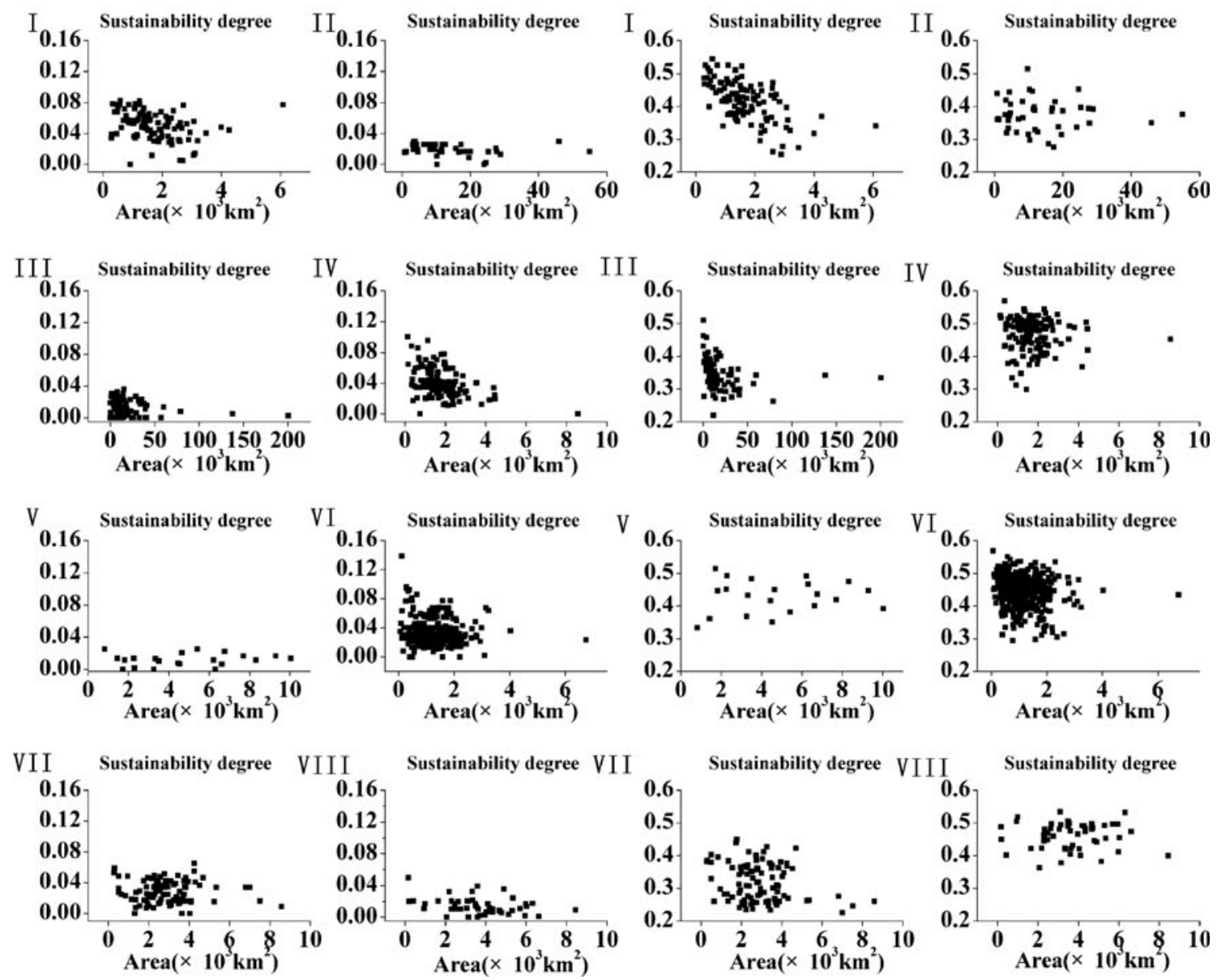
Fig. 2 Scale effects on sustainability level at regional (A) and county (B) levels by two different ways of measuring sustainability (a focusing on balanced performance; $\mathbf{b}$ focusing on aggregate achievement) (A) sustainability level versus county area (top) and sustainability level versus number of counties (bottom). Green represents environmental sustainability, red represents economic sustainability, magenta represents social sustainability, and blue represents comprehensive sustainability. B Sustainability level versus county area in each region. The identification of agricultural regions refers to Fig. 1

methods were selected: equal weighting, principal component analysis, and Analytic Hierarchy Processing. Figure $3 \mathrm{~b}$ shows the rankings among regions produced by these three weighting methods. The degree of sustainability based on principal component analysis and equal weighting showed a Spearman correlation coefficient $(r)=0.86^{* *}$, and the Spearman correlation coefficient was lower between equal weighting and Analytic Hierarchy Processing, $(r)=0.83^{*}$. The weighting methods of principal component analysis and equal weighting were found to have roughly the same impact on the regional ranking except for Xinjiang and South China. Analytic Hierarchy Processing is preferred because scholar opinions were involved in the weighting process.

The impact of aggregation methods including all indicators on ranking among regions was finally evaluated. Three aggregation methods were selected: additive aggregation, geometric aggregation, and minimum aggregation, representing full compensation, partial compensation, and non-compensation between indicators, respectively. Different regions showed different levels of sensitivity to the choice of aggregation method. Regions at the top left of Fig. 3c showed relatively good performance when allowed compensating for their deficiencies in some indicators under the additive aggregation method. These included the Songnen Plain, Sanjing Plain, and Xinjiang. Middle reaches of the Yangtze River and Jianghuai region and Eastern Inner Mongolia showed less sensitivity to the aggregation method. Figure $3 \mathrm{~d}$ shows the full compensation method versus partial compensation method. An apparent feature is that most sustainable regions were affected by aggregation methods to a more extent.

Fig. 3 Sensitive analysis on region ranking according to sustainability level. a Additive-based ranking of 15 scenarios with indicator selection. Black lines correspond to the median of the simulated ranks. Whiskers show the best and worst ranks across 15 scenarios produced either by considering all 14 indicators or by excluding one indicator at a time. b Region ranking based on three weighting methods: principal component analysis (red), Analytic Hierarchy Processing (green), and equal weighting (blue). $\mathbf{c}$ Region ranking based on different aggregation methods (minimum aggregation versus additive aggregation). d Region ranking based on different aggregation methods (geometric aggregation versus additive aggregation). The identification of agricultural regions refers to Fig. 1

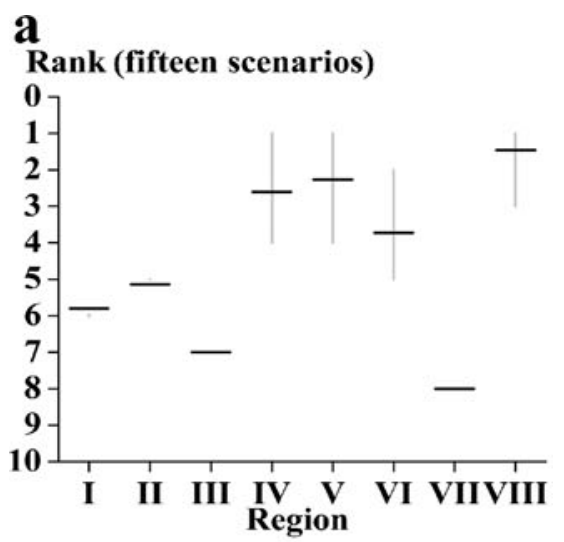

b

Rank (three weighting methods)

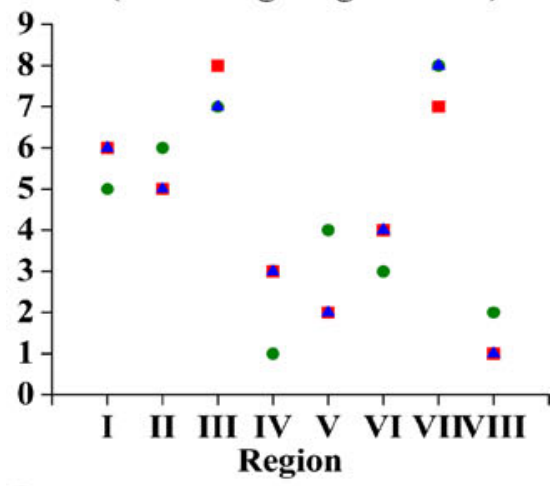

c

Additive aggregation rank

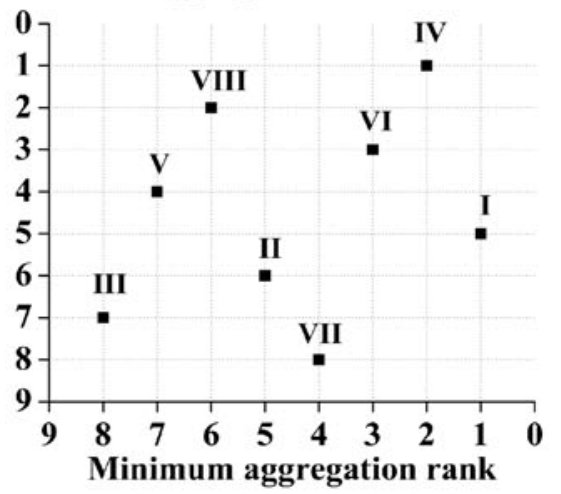

d

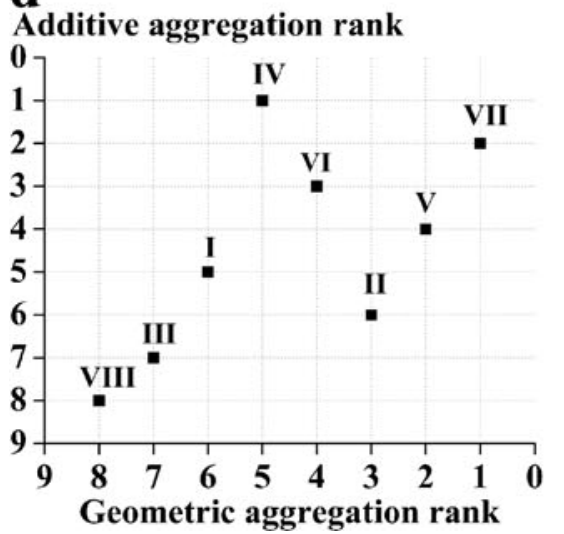




\section{Conclusion}

In order to facilitate overall understanding of sustainability in agricultural regions throughout China with a detailed spatial variability at finer scales, sustainability assessment was conducted in China's eight major agricultural regions at the county level. Restraints were identified in each region, which may allow regional managers to make better decisions.

Based on two methods of measuring sustainability, this paper proposed a methodological framework for the assessment and comparison of land use sustainability on a regional scale integrating three pillars: environment, economy, and society. An indexing system for sustainability assessment within a large-scale geographic domain was developed for identification of the sustainability indicators, capturing significant information about land use systems. This could provide reference information for the construction of sustainability assessment framework at the regional scale.

A significant difference in level of sustainability (environmental, economic, social, and comprehensive) across eight regions for two methods (method I (method II)): 0.06-0.57 (0.47-0.87), 0.01-0.06 (0.11-0.42), 0.04-0.10 (0.14-0.31), and $0.01-0.05(0.31-0.46)$. Region ranking of sustainability level varied with different methods of measuring sustainability, which wasn't addressed in the previous studies. When evaluated using the method focusing on the balanced performance among different aspects of sustainability, Xinjiang was found to be the least sustainable region (i.e., 0.01) because of poor land quality and serious land degradation, and the Sichuan Basin was found to be the most one (i.e., 0.05). When evaluated using the method indicating aggregate achievement among indicators, the results were significantly different: Middle reaches of the Yangtze River and Jianghuai region were found to be the most sustainable (i.e., 0.46), and South China was found to be the least one (i.e., 0.31) because of the large income gap between urban and rural areas. Sensitivity analysis showed Songnen and Sanjiang Plains were more sensitive to the indicator selection and aggregation rules. Scale effects didn't exist in sustainability assessment at the regional level.

Acknowledgments This study has been funded by the National Natural Science Foundation of China (no. 41071065). The authors thank Lanhai Li Professor and Yang Yang for their valuable and constructive comments on the manuscript.

\section{References}

Andreoli M, Tellarini V (2000) Farm sustainability evaluation: methodology and practice. Agric Ecosyst Environ 77:43-52. doi:10.1016/S0167-8809(99)00091-2

Barrett CB (2010) Measuring food insecurity. Science 327:825-828. doi: $10.1126 /$ science. 1182768
Bell S, Morse S (2008) Sustainability indicators: measuring the immeasurable? Earthscan, London

Bockstaller C, Girardin P (2003) How to validate environmental indicators. Agric Syst 76:639-653. doi:10.1016/S0308-521X (02)00053-7

Boyd DR (2001) Canada vs. the OECD: An Environmental Comparison. EcoResearch Law and Policy. University of Victoria, http://www.environmentalindicators.com/htdocs/PDF/ CanadavsOECD.pdf. Accessed 10 May 2012

Cai Y, Barry S (1994) Sustainability in agriculture: a general review. Agric Ecosyst Environ 49:299-307. doi:10.1016/0167-8809 (94)90059-0

Dantsis T, Douma C, Giourga C et al (2010) A methodological approach to assess and compare the sustainability level of agricultural plant production systems. Ecol Indic 10:256-263. doi:10.1016/j.ecolind.2009.05.007

Godfray HCJ, Beddington JR, Crute IR et al (2010) Food security: the challenge of feeding 9 billion people. Science 327:812-818. doi:10.1126/science. 1185383

Gómez-Limón JA, Riesgo L (2009) Alternative approaches to the construction of a comprehensive indicator of agricultural sustainability: an application to irrigated agriculture in the Duero basin in Spain. J Environ Manag 90:3345-3362. doi:10.1016/j.jenvman.2009.05.023

Guitouni A, Martel JM (1998) Tentative guidelines to help choosing an appropriate MCDA method. Eur J Oper Res 109:501-521

Jiang L, Tong Y, Zhao Z et al. (2003) Impacts of population migration on land degradation in Tarim River Basin, Xinjiang of China. Paper presented at the Annual Meeting of the Population Association of America, May 1-3, Minneapolis, US

Jin S (1983) The application of regression equation in the determination of reasonable fertilizer application amount. Gansu Agric Sci Technol 11:9-13 (in Chinese)

Kong W, Ou M (2006) Study on the state of cultivated land and its sustainable use in Shandong province. China Popul, Resour Environ 16:44-48 (in Chinese)

Liu J, Liu M, Tian H et al (2005) Spatial and temporal patterns of China's cropland during 1990-2000: an analysis based on Landsat TM data. Remote Sens Environ 98:442-456. doi:10.1016/j.rse.2005.08.012

Liu D, Wang Z, Song K et al (2009) Land use/cover changes and environmental consequences in Songnen Plain, Northeast China. Chinese Geogr Sci 19:299-305. doi:10.1007/s11769009-0299-2

Malczewski J (2006) GIS-based multicriteria decision analysis: a survey of the literature. Int J Geogr Inf Sci 20:703-726

Meul M, Van Passel S, Nevens F et al (2008) MOTIFS: a monitoring tool for integrated farm sustainability. Agron Sustain Dev 28:321332. doi:10.1051/agro:2008001

Meul M, Nevens F, Reheul D (2009) Validating sustainability indicators: focus on ecological aspects of Flemish dairy farms. Ecol Indic 9:284-295. doi:10.1016/j.ecolind.2008.05.007

Nardo M, Saisana M, Saltelli A et al. (2008) Handbook on constructing comprehensive indicators: methodology and user guide. OECD Statistics Working Paper 2005/3, OECD Publishing. doi:10.1787/ 18152031

National Agricultural Regionalization Commission (1981) China's comprehensive agricultural regionalization. Agricultural Press, Beijing, in Chinese

Roy R, Chan NW (2012) An assessment of agricultural sustainability indicators in Bangladesh: review and synthesis. Environmentalist 32:99-110. doi:10.1007/s10669-011-9364-3

Shu G, Zhou YX, Zhang MH et al (2001) A sustainable agroecological solution to water shortage in the North China Plain (Huabei Plain). J Environ Plan Manage 44:345-355. doi:10.1080/ 09640560120046106 
Tao F, Yokozawa M, Liu J et al (2009) Climate change, land use change, and China's food security in the twenty-first century: an integrated perspective. Clim Chang 93:433-445. doi:10.1007/ s10584-008-9491-0

TexaSoft (2011) WINKS SDA Software, vol 7. TexaSoft, Cedar Hill

Van Cauwenbergh N, Biala K, Bielders C et al (2007) SAFE-a hierarchical framework for assessing the sustainability of agricultural systems. Agric Ecosyst Environ 120:229-242. doi:10.1016/ j.agee.2006.09.006
Van Passel S, Nevens F, Mathijs E et al (2007) Measuring farm sustainability and explaining differences in sustainable efficiency. Ecol Econ 62:149-161. doi:10.1016/j.ecolecon.2006.06.008

Wu K, Huang R (2001) The sustainable evaluations, the development potentialities and the countermeasures of water and land resources use in the Huang-Huai-Hai Plain. Sci Geogr Sin 21:390-395 (in Chinese)

Yang G (2001) The process and driving forces of change in arable-land area in the Yangtze River Delta during the past 50 years. J Nat Resour 16:121-127 (in Chinese) 